\title{
A short telephone-call reminder improves bowel preparation, quality indicators and patient satisfaction with first colonoscopy
}

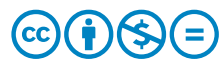

\author{
Authors \\ Marisol Gálvez, Angel Mario Zarate, Hector Espino, Fátima Higuera-de la Tijera, Richard Alexander Awad, Santiago \\ Camacho
}

Institution

Endoscopy Unit of Gastroenterology Service, Mexico City

General Hospital “Dr Eduardo Liceaga”, Mexico City, Mexico

submitted 18.5 .2017

accepted after revision 3.7.2017

\author{
Bibliography \\ DOI https://doi.org/10.1055/s-0043-117954 | \\ Endoscopy International Open 2017; 05: E1172-E1178 \\ (c) Georg Thieme Verlag KG Stuttgart · New York \\ ISSN 2364-3722
}

Corresponding author

Santiago Camacho, Gastroenterology Service, Mexico City General Hospital “Dr Eduardo Liceaga”, Dr. Balmis No 148, Col. Doctores CP 06720, Delegación Cuauhtémoc, Mexico City, Mexico

Fax: (+5255) 27892000

santiach@yahoo.com.mx

\section{ABSTRACT}

Background and study aims Addition of a reminder program to conventional indications improves colonoscopy. The aim of this study was to evaluate the effectiveness of a short telephone call reminder (STCR) on a patient's first colonoscopy.
Patients and methods One day before colonoscopy, we made a STCR of $<10$ minutes to 141 randomly selected patients of 258 recruited. The STCRs informed patients about the procedure date, indications for taking laxatives, and dietetic requirements. Questions were clarified only when patients asked directly. We evaluated bowel preparation, quality indicators, and patient satisfaction. Data were expressed as mean \pm SD and percentages. Statistical differences were evaluated by Student's $t$ and Chi squared tests; alpha $=0.05$. All authors had access to the study data and reviewed and approved the final manuscript.

Results The STCR group had better bowel preparation which was demonstrated by higher completion frequency ( $97.16 \%$ vs. $82.05 \%)$, in less time $(4.52 \pm 3.06$ vs. $5.38 \pm$ 3.03 hours) intake of laxative, and higher Boston's scale (7.66 \pm 2.42 vs. $5.2 \pm 1.65)$. Quality indicators of colonoscopy were better in patients that received a STCR [cecal intubation rate: $100.00 \%$ vs. $87.18 \%$; polyp detection: $42.55 \%$ vs. $9.4 \%$; and cecal arrival time (min): $12.09 \pm 3.62$ vs. $15.09 \pm 5.02$ ]. STCR patients were more satisfied $(97.87 \%$ vs. $55.56 \%)$ and would repeat colonoscopy (21.99\% vs. $11.11 \%)$.

Conclusions A simple additional step such as a STCR improves quality of bowel preparation, quality indicators, and satisfaction of patients undergoing their first colonoscopy. Clinical trial registry in Mexico City General Hospital: DI/16/ $107 / 3 / 108$.

\section{Introduction}

Colonoscopy is the gold standard of colorectal cancer screening [1], but as high as $9 \%$ of colorectal carcinomas are made on the latter vigilance [2]. Adequate detection of lesions during the first colonoscopy is essential to make an early diagnosis, reduce the incidence of colorectal cancer and therefore be cost-effective.

Adequate colonoscopy depends on performomg quality indicators adequately (cecal intubation, arrival and withdraw time, and polyp detection) [3]. These quality indicators are di- rectly related to bowel preparation (BP) which can be suboptimal in up to $30 \%$ of patients [4]. When BP is poor, adenoma detection is less effective [5] and costs increase by $12 \%$ to $22 \%$ [6]. Compared with excellent BP, poor BP is a factor associated with recommendation of a 3 -year vs a 5 -year surveillance interval (RR 2.16) [7].

$\mathrm{BP}$ is a bothersome procedure associated with personal barriers that include anxiety, worry about anticipated pain, and complications inherent with the procedure [8]. About $20 \%$ of patients with failed BP do not follow instructions satisfactorily [9] and that is the main factor for poor BP [10]. Physician ad- 
vice, patient medical relationship, concern for colon cancer and adequate communication are factors that significant influence procedure acceptance and therefore better adherence to instruction prior to the procedure [11].

There are programs that incorporate elements aimed at reducing fear and anxiety and helping to ensure patient understanding of written material, and they ensuring that all patients receive the same information and follow proper instructions that improve the quality of BP for colonoscopy [10]. Such programs include counseling sessions, educational booklets, visual aids and educational videos to improve patient comprehension as well as reminders to patients about the details of BP instructions through Short Messenger Service (SMS) and telephone calls [12]. Use of a telephone call 1 day before screening improves BP and reduces incidence of non-compliance with instructions [9].

These programs, however, consume time and human resources and therefore may be difficult to implement in high-demand units. Our hypothesis is that a Short Telephone Call Reminder (STCR) using a predetermined script enhances the first colonoscopy. The objective of this study was to evaluate the effectiveness of a STCR ( $<10$ minutes) on BP, quality indicators and patient satisfaction with the first colonoscopy procedure.

\section{Patients and methods}

\section{Subjects}

This clinical randomized, single-blind controlled trial was conducted at the Endoscopy Unit of Gastroenterology Service in the Mexico City General Hospital and in accordance with the Declaration of Helsinki and its subsequent revisions [13]. Local research and ethical committees approved the protocol (DI/16/ $107 / 3 / 108)$. Informed consent of all subjects prior the procedure was obtained. All consecutive outpatients presenting for first colonoscopy study from January to May 2016 were recruited. To participate, patients needed to be older than 18 years, scheduled for first colonoscopy and to give consent to the study. Patients with previous colonoscopy, history of colorectal surgery and/or colonic stenosis, suspected megacolon, toxic colitis or intestinal obstruction, severe heart failure, renal failure, or who had no phone were excluded. Patients who expressed their desire to not participate in the study, who were unable to be contacted and/or failed to understand standard instructions were ineligible.

\section{Study protocol}

On the day of programming the study all patients were given a standard explanation that included ingestion of a single dose of $4 \mathrm{~L}$ of laxative (Nulytely ${ }^{\mathrm{TM}}$, Asofarma. Composition: $105 \mathrm{~g}$ of Polyethylene glycol, $1.43 \mathrm{~g}$ of sodium bicarbonate, $2.8 \mathrm{~g}$ of sodium chloride and $0.37 \mathrm{~g}$ of potassium chloride). We instructed patients to reconstitute an envelope of powdered Nulytely ${ }^{\mathrm{TM}}$ in $1 \mathrm{~L}$ of lukewarm water starting at 17:00 hours the day before colonoscopy and to drink the contents within 1 hour (approximately $250 \mathrm{~mL}$ each 15 minutes). We asked them to repeat this instruction 3 more times to complete $4 \mathrm{~L}$ to finish around 21:00 hours. They were also given instructions to consume a liquid diet the day before the study. Medical or nursing staff provided information to all patients in the form of brochures and leaflets, which were explained to the patients and taken home.

We asked all patients for at least 1 phone number at which we could call them. They were warned that only some of them would be called. All patients agreed, as part of the study protocol; that they did not know which group they would belong to. We select patients previously randomized to make a STCR or not, one day before the study. The STCR was made by MGM with the following standard script: "Good morning, I am calling from the endoscopic unit of the Mexico City General Hospital with a reminder about your study, which is scheduled for tomorrow at 8:00 AM. You must initiate laxative intake at 5:00 PM. Dissolve 1 pack of Nulytely in $1 \mathrm{~L}$ of water and take all contents every hour to complete $4 \mathrm{~L}$ pack. Your diet must be liquid consisting of juices without waste or pulp, tea, beef broth and/or chicken with vegetables, no meat, water ad libitum, avoid consuming red drinks, red jelly, fruits, vegetables and tortillas. Thank you for taking this call. We are looking forward to seeing you tomorrow." The reading time of this text lasts approximately 1 minute and allows questions at the end of the call. Questions were clarified only when patients asked directly. All calls were made in less than 10 minutes per patient and all telephone sessions took no more than 30 minutes and were done from Sunday to Thursday between 12:00 and 12:30.

On colonoscopy day, before the procedure each patient answered a questionnaire about the quantity and duration of laxative intake as well any adverse events (AEs) related to it. The colonoscopy was made in a standardized form and the personnel was blinded to patient group. To avoid the "Hawthorne effect" [14], the endoscopists were unaware if a patient belonged to this study or was part of their normal clinical practice. Each endoscopist's competency (MGM, AMZG and HEC) was certificated by the Mexican Council of Gastroenterology [15], which takes into account ASGE guidelines [16]. All procedures were performed by a board-certificated physician or a resident in training under physician supervision and with an anesthesiologist. Anesthetics were used according to international recommendations and drug use was modified according to anesthesiologist criteria. There were no serious AEs or complications associated with any of the procedures. At the end of the study each patient was asked to rate satisfaction with the procedure using a 4-point Likert-scale (unsatisfied, somewhat satisfied, satisfied or very satisfied) and to indicate whether he or she would repeat the study.

\section{Colonoscopy quality standards}

We evaluated colonoscopy quality standards based on international guidelines from ASGE and ACG [17]. These guidelines take into account cecal intubation, time to get to the cecum, time of withdrawal, and polyp detection. To measure bowel cleansing we used the Boston Bowel Preparation Scale (BBPS) [18]. It separates the colon into 3 segments - right, transverse, and left colon - assigning a score from 0 (non-prepared colon, mucosal not visualized with solid feces) to 3 (excellent mucosal visualization, without liquid feces) in each segment. Scores went from 0 (unprepared bowel) to 9 (excellent preparation). 
Clinically a score $>5$ was considered adequate bowel preparation because it is associated with higher rates of polyp detection [19].

\section{Sample size calculation}

A sample size calculation was performed with StatMate 2 for windows (GraphPad Software Inc). According to a study by Liu $X$ [9], adequate BP was achieved in $81.6 \%$ patients who received telephone re-education a day before colonoscopy vs $70.3 \%$ of controls. To detect the difference with a significance level $(\alpha)$ of 0.05 and a power of $80 \%$ with a chi-square test and assuming a $11 \%$ difference in the rate of colonic cleansing in a BBPS $>5$, we calculated that we needed 150 patients per group.

\section{Statistical analysis}

The main outcome was BBPS in both groups. Secondary outcome variables were colonoscopy quality standards and satisfaction with the procedure. Other variables included indications for colonoscopy, diagnosis, histopathological findings, age, sex, years of formal study (scholarship), waiting time for colonoscopy, duration and amount of laxative intake, concomitant chronic degenerative diseases (diabetes mellitus and/or systemic arterial hypertension), family history of colorectal cancer (first and no-first relative), smoking (history and current) and collateral effects. Data were entered in an electronic sheet (Excel 2016, Microsoft). Statistical analysis was done with Prims 3.0 (Graph Pad software, Inc). Data were expressed according to mean \pm standard deviation (SD), $95 \%$ confidence intervals $(\mathrm{Cl})$, and percentages when appropriate. Statistical differences were evaluated by Student's $t$ test, Chi squared tests and Fisher's Exact Test with Katz approximation for relative risk (RR); alpha $=0.05$.

\section{Results}

\section{Population}

We prospectively recruited 300 patients and excluded $14 \%$ of patients because they did not undergo colonoscopy; of them, $9(3 \%)$ were in the STCR group and $37(11 \%)$ were in the noSTCR group. The RR for not undergoing colonoscopy for STCR and no-STCR was $1.369(P<0.001)$. A STCR was made to 141 (54.65\%) randomized patients of 258 patients recruited. All procedures were done in the morning (10:56 $\pm 2: 59$ hours) and about 14 hours after the last laxative dosage.

There were no differences in age $[51.97 \pm 14.78$ (49.53$54.41)$ vs. $51.16 \pm 15.88(48.29-54.04)$ years, $P=0.67]$, sex (63.12\% vs.68.38\% females, $P=0.43$ ), scholarship [7.66 \pm 3.23 $(7.12-8.19)$ vs. $7.69 \pm 3.13(7.13-8.26)$ years, $P=0.92]$, days waiting for the procedure $[42.32 \pm 21.07(38.84-45.8)$ vs. $41.03 \pm 22.67(36.92-45.13)$ days, $P=0.63]$, prevalence of concomitant chronic and degenerative diseases $(29.79 \%$ vs. $27.35 \%, P=0.76)$, colorectal cancer antecedents $(10.64 \%$ vs. $6.84 \%, P=0.24)$ or smoking habits $(11.35 \%$ vs. $10.26 \%, P=$ 0.23 ) between the STCR vs. no-STCR groups; respectively.

\section{Bowel preparation}

STCR group had better BP demonstrated by shorter intake and higher frequency of completion of laxative; and higher Boston's scale in each segment ( $\triangleright$ Table 1$)$. BBP $\leq 5$ were less frequent in patients in the STCR versus the no-STCR group (1\% vs $49 \%$, respectively, $P<0.001)$ with a $R R$ of $2.24(P<0.0001)$. Collateral effects were similar between groups. In patients with very poor bowel cleansing we did not use any other technique to improve colonoscopy, we classified the study as incomplete, and resche-

- Table 1 Bowel preparation.

\begin{tabular}{|l|l|c|}
\hline & $\begin{array}{l}\text { SCTR group } \\
\text { (n=141) }\end{array}$ & $\begin{array}{l}\text { No SCTR group } \\
\text { (n=117) }\end{array}$ \\
\hline Laxative intake total time (Hrs:min) & $4: 52 \pm 0: 52(4: 44-5: 01)$ & $5: 38 \pm 1: 25(5: 23-5: 54)$ \\
\hline Full Laxative intake & $97.16 \%$ & $82.05 \%$ \\
\hline Boston Scale & & \\
\hline - Right colon & $2.49 \pm 0.55(2.4-2.58)$ & $1.7 \pm 0.53(1.6-1.8)$ \\
\hline - Transverse colon & $2.62 \pm 0.49(2.54-2.7)$ & $1.83 \pm 0.44(1.75-1.91)$ \\
\hline - Left colon & $2.56 \pm 0.51(2.48-2.64)$ & $1.85 \pm 0.53(1.76-1.95)$ \\
\hline - Total score & $7.66 \pm 0.82(7.52-7.79)$ & $5.2 \pm 1.27(4.97-5.43)$ \\
\hline Collateral effect & & \\
\hline - Pain & $14.18 \%$ & $14.53 \%$ \\
\hline - Nausea & $16.31 \%$ & $10.26 \%$ \\
\hline - Vomiting & $1.42 \%$ & $5.98 \%$ \\
\hline - None & $68.09 \%$ & $69.23 \%$ \\
\hline
\end{tabular}


- Table 2 Colonoscopy quality standards.

\begin{tabular}{|l|c|c|c|}
\hline & SCTR group $(\mathbf{n = 1 4 1 )}$ & No SCTR group (n=117) & $P^{*}$ \\
\hline Cecal intubation & $100.00 \%$ & $87.18 \%$ & $<0.0001$ \\
\hline Polyp detection & $42.55 \%$ & $9.40 \%$ & $<0.0001$ \\
\hline Cecum arrival time $(\mathrm{min})$ & $12.09 \pm 3.62(11.49-12.69)$ & $15.09 \pm 5.02(14.14-16.05)$ & $<0.0001$ \\
\hline Withdrawal time $(\mathrm{min})$ & $9.9 \pm 2.96(9.41-10.39)$ & $10.65 \pm 3.33(10.05-11.25)$ & 0.0570 \\
\hline $\begin{array}{l}\text { Data express percentage and mean } \pm \text { SD }(95 \% \text { Cl) } \\
\text { * wCTR group vs. No SCTR group. Exact Chi-square test, two sided or Non-Paired Student } t \text { Test, two sided }\end{array}$
\end{tabular}

- Table 3 Indications for colonoscopy.

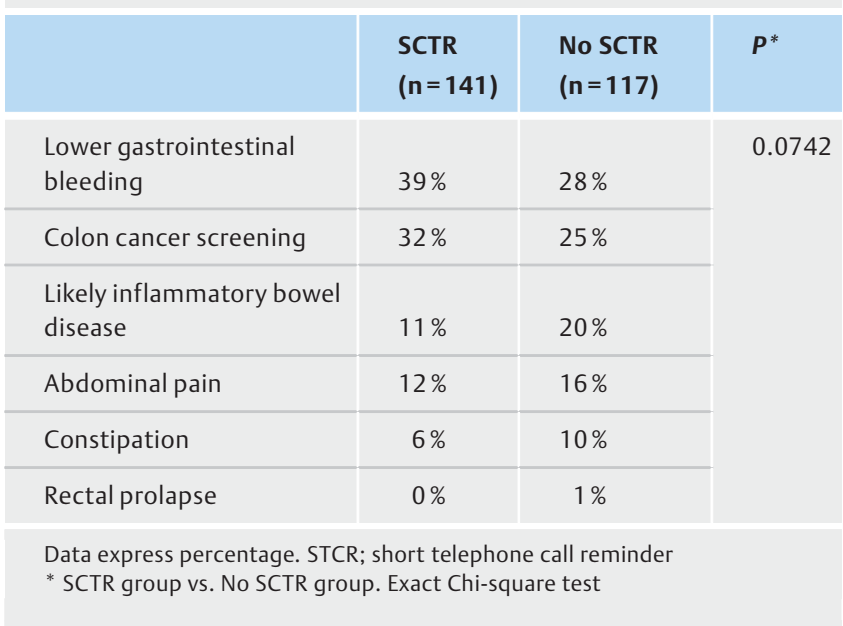

dule the procedure for the following month according to administrative procedures.

\section{Colonoscopy quality standards}

The quality indicators for colonoscopy differed in patients with STCR, except for withdrawal time ( $\triangleright$ Table 2). The RR for polyp detection was $4.52(P<0.0001)$ between the SCTR $(42.5 \%)$ and the no-SCTR $(9.4 \%)$ groups.

\section{Indications for colonoscopy}

Indications for colonoscopy are shown in $>$ Table 3 and did not differ between the two groups. The two main indications for first colonoscopy were lower gastrointestinal bleeding and screening for colorectal cancer.

\section{Colonoscopy findings and satisfaction}

Diagnoses differed between groups ( $>$ Table 4$)$. The RR for incomplete colonoscopies was $1.182(P<0.0001)$ between the STCR group (none) vs. the no-STCR group (18 patients, $15 \%$ ). STCR patients were more satisfied $(97.87 \%$ vs. $55.56 \%, P<$ $0.001)$ and more likely to repeat colonoscopy $(21.99 \%$ vs. $11.11 \%, P=0.0043)$.
Table 4 Colonoscopy findings.

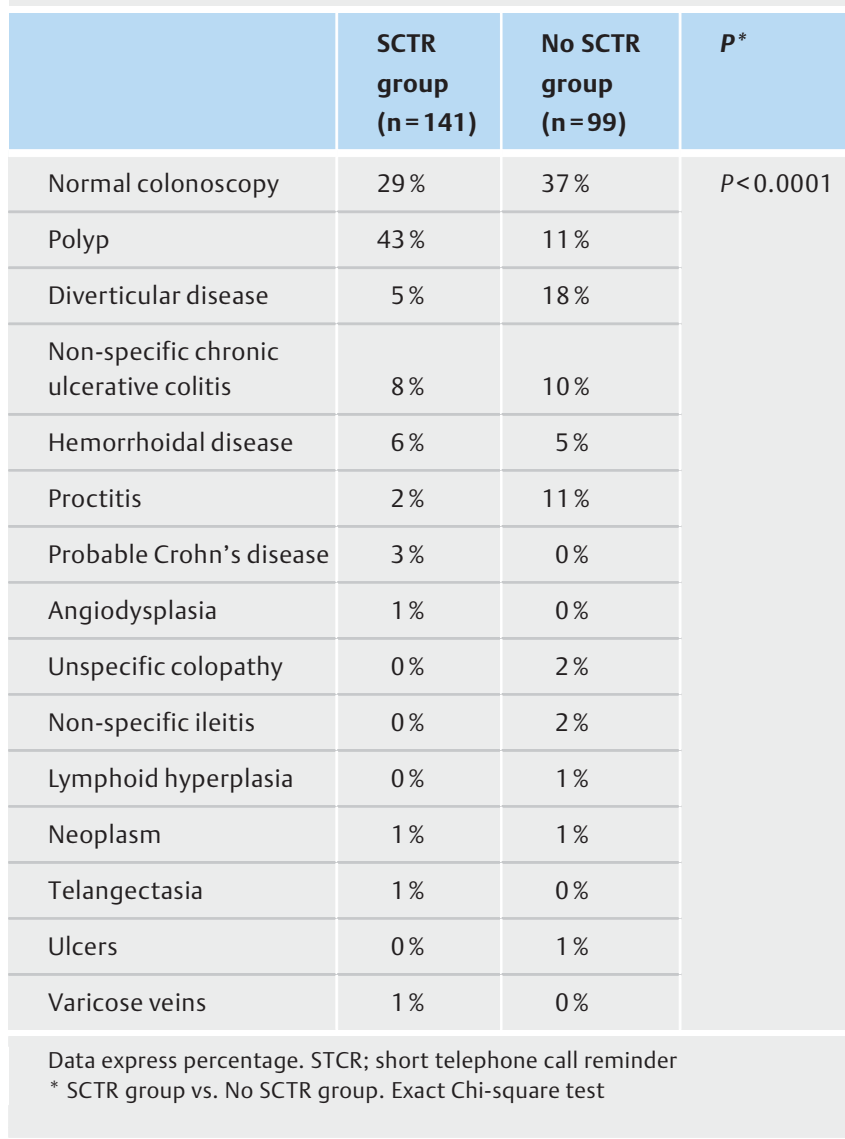

\section{Polyps histopathology}

Of the 71 patients with polyps, 60(42.55\%) were in the STCR group and $11(9.4 \%)$ in the no-STCR group. Three reports were lost because of administrative errors, thus we examined 68 histopathology findings ( $>$ Table 5 ). The adenoma detection rate (ADR) was higher in the STCR group (24\%) than in the no-STCR group (3\%). The RR for ADR lesions was $1.85(P<0.001)$ between the STCR group and no-STCR group.

\section{Discussion}

This study shows that a STCR a day before first colonoscopy improves patient attendance, BP, quality standards for the procedure and patient satisfaction. 
- Table 5 Polyps histopathology findings.

\begin{tabular}{|c|c|c|c|}
\hline & $\begin{array}{l}\text { SCTR } \\
\text { group } \\
(n=57)\end{array}$ & $\begin{array}{l}\text { No SCTR } \\
\text { group } \\
(n=11)\end{array}$ & $P^{*}$ \\
\hline \multicolumn{4}{|l|}{ Neoplastic finding } \\
\hline - Tubular adenoma & $36 \%$ & $30 \%$ & \multirow[t]{5}{*}{0.9512} \\
\hline - Tubulovillous adenoma & $12 \%$ & $10 \%$ & \\
\hline - Villous adenoma & $5 \%$ & $0 \%$ & \\
\hline - Adenocarcinoma & $2 \%$ & $0 \%$ & \\
\hline - Serrated polyp & $2 \%$ & $0 \%$ & \\
\hline \multicolumn{4}{|l|}{ Non-neoplastic finding } \\
\hline - Hyperplastic polyp & $31 \%$ & $40 \%$ & \multirow[t]{5}{*}{0.1452} \\
\hline - Inflammatory polyp & $7 \%$ & $0 \%$ & \\
\hline - Hamartomatous polyp & $2 \%$ & $10 \%$ & \\
\hline - Proctitis & $3 \%$ & $0 \%$ & \\
\hline - Juvenile polyp & $0 \%$ & $10 \%$ & \\
\hline
\end{tabular}

Rates of non-attendance to undergo for a colonoscopy (4\% to $25 \%$ ) [20] are so serious that had have developed programs to help prevent these absences, [21] including telephone calls [22]. In our study, STCR reduced the risk of not presenting at the first colonoscopy by $20 \%$, suggesting the need to establish the STCR as a standard protocol for all colonoscopies.

Lower levels of education (OR:2.35), longer waiting times (OR:1.86) and noncompliance with instructions (OR:4.76) are risk factors associated with poor BP [23]. It is believed that patients with less education have less understanding of the importance of BP. Despite that, there were no differences in education levels between our groups, so we infer that STCR is useful independent of patient education level. Because instructions may be forgotten, longer waiting times for an appointment are associated with bad BP. In our study, the waiting time was typically of 5 weeks, with no differences between groups. Between $18 \%$ and $23.5 \%$ of patients do not follow instructions and therefore they do not accomplish adequate BP [24]. Our STCR group had better BP as demonstrated by more frequent complete intake of laxative in less time. That is in keeping with findings that lack of adherence to BP indication and incorrect timing of laxative intake are associated with reduced BP quality [9]. Collateral effects were similar in both groups.

The STCR group had better BBPS. A BBPS $<6$ correlates with a recommended time to repeat colonoscopy of $<1$ year and had less correlation with insertion and withdrawal times [18]. A BBPS $>6$ is defined as adequate for a 10-year interval for follow-up colonoscopy [25]. The STCR group had higher rates of cecum intubation and less time to arrival with no difference in withdrawal times. When BP is poor, colonoscopy takes longer, is more difficult and has a lower rate of cecal intubation and is more bothersome for patients [23]. Cecum cannulation was achieved in $93 \%$ of our patients, which is a slightly higher than reported rates of $90 \%$ [26]. Cecum cannulation was done in all STRC patients compared to $87.18 \%$ of no-STRC group. Withdrawal time should last a minimum of 6 minutes to ensure good examination [27] and more than 9 minutes for high-quality colonoscopy [28]. In our study, withdrawal times (>9 minutes) were similar in both groups.

The polyp detection rate was better in the STCR group. In our STCR group, rates of polyp detection were similar to those seen in studies in Italy (35\%), Spain (45.8\%), the United States (49\%), France $(35.5 \%)$ and Iran (23.5\%), and they were much higher than in our no-STCR group (9.4\%) [29]. Geographical and cultural variations, however, are important determinants of frequency of detection of polyps in a general population. To our knowledge ours is the first report of polyp detection by colonoscopy in the adult Mexican population.

Adequate ADR is essential to make an early diagnosis and reduce incidence of colorectal cancer, and therefore, ensure costeffectiveness [1]. When BP is poor, adenoma is found on subsequent colonoscopy within 3 years in between $28 \%$ and $42 \%$ of patients [30]. The total ADR in our population was 14.3, similar the 14.69 reported previously [31]. In our study, the ADR was higher in the STCR group than in our no-STCR group. This is the first report of ADR in our hospital.

Lower gastrointestinal bleeding and screening for colorectal cancer were the main indications for colonoscopy in our study which is consistent with colonoscopies performed around the world [29]. Our study shows that a no-STCR group has a RR of 1.82 for an incomplete colonoscopy.

Data are conflicting on use of patient satisfaction as a quality measure for colonoscopy, with one study finding no correlation with quality metrics [32] and another study finding that higher cecal intubation rates improve patient comfort [33]. Besides this consideration, the European Society of Gastrointestinal Endoscopy suggests using patient satisfaction as a quality indicator for gastrointestinal endoscopy [34]. Patients in our STCR group were more satisfied and would repeat colonoscopy. Given that evidence, we agree that patient satisfaction should be a goal for quality endoscopy because a satisfied patient may recommend that other individuals have the procedure performed, increasing the likelihood that eligible patients will be screened [35].

There are only 2 studies with BP reinforcement by telephone [9] and SMS [36] that reported significantly increased quality of BP. One study showed that use of SMS and a telephone call 2 days prior to colonoscopy improved BP in both groups compared with controls [36]. Although the authors concluded that SMS was more cost-effective than a telephone call, its use has several limitations: the need for a significant technological and investment base [37]; inability to exchange information with a patient, which does not allow for proper exercise of patientcentered medicine with shared-decision [38]; and potentially increased anxiety in patients who do not use SMS regularly. Prior training with SMS also is necessary. Our methodology requires only reading a script and therefore is easier to implement. 
Our study has several limitations. Although split preparation is best for BP [10], we choose a single dose. Our patients did not want to take the laxative 2 hours before colonoscopy because they were afraid of soiling since travel on public transportation was required. Also a report in our hospital has found no difference in colonic cleansing between use of a split preparation versus a single dose of laxative [39]. We lost $14 \%$ of patients because they did not keep their colonoscopy appointments. Although our unit performs on average 6 to 8 colonoscopies per day, we recruited only 3 patients per day for first colonoscopy because we believe that a patient's previous subjective perception and BP training would influence the protocol. We do not know if STCR improves preparation in patients who have already undergone colonoscopy and it is the subject of another protocol. Colonoscopy can be highly variable because it is operator-dependent. To standardize it, each procedure was validated by a certified endoscopist who also was a professor according to national [15] and international standards [16]. Physician training is a frequent activity in academic and research units. We decided to measure the effectiveness of STCR without altering the rhythm, participation or demand for the service. We did not follow patients over the medium or long term, which makes it impossible to know the frequency of repeat colonoscopies at 1,3 , and 5 years, however, that was not the objective of this study. We also did not measure sedation used, although it was standardized and it was the anesthesiologist's decision whether to to increase or decrease anesthesia dosage. Sedation is an important factor in patient satisfaction and yet none of our patients had AEs or complications during their procedures.

\section{Conclusion}

In conclusion, a script-based STCR increases first colonoscopy quality and improves patient attendance at the procedure and satisfaction with it. We suggest the need to establish a telephone call as a standard protocol for all first-time colonoscopies.

\section{Competing interests}

None

\section{References}

[1] Brand EC, Wallace MB. Strategies to Increase Adenoma Detection Rates. Curr Treat Options Gastroenterol 2017; 15: 184-212

[2] Benedict M, Galvao NA, Zhang X. Interval colorectal carcinoma: An unsolved debate. World J Gastroenterol 2015; 21: 12735-12741

[3] Jang JY, Chun HJ. Bowel preparations as quality indicators for colonoscopy. World J Gastroenterol 2014; 20: 2746-2750

[4] Serper M, Gawron AJ, Smith SG et al. Patient factors that affect quality of colonoscopy preparation. Clin Gastroenterol Hepatol 2014; 12: $451-457$
[5] Rex DK, Johnson DA, Anderson JC et al. American College of Gastroenterology guidelines for colorectal cancer screening 2009 [corrected]. Am J Gastroenterol 2009; 104: 739 - 750

[6] Rex DK, Imperiale TF, Latinovich DR et al. Impact of bowel preparation on efficiency and cost of colonoscopy. Am J Gastroenterol 2002; 97: $1696-1700$

[7] Anderson JC, Baron JA, Ahnen DJ et al. Factors associated with shorter colonoscopy surveillance intervals for patients with low-risk colorectal adenomas and effects on outcome. Gastroenterology 2017; 152: $1933-1943 . e 5$

[8] McLachlan SA, Clements A, Austoker ]. Patients' experiences and reported barriers to colonoscopy in the screening context - a systematic review of the literature. Patient Educ Couns 2012; 86: 137- 146

[9] Liu X, Luo H, Zhang L et al. Telephone-based re-education on the day before colonoscopy improves the quality of bowel preparation and the polyp detection rate: a prospective, colonoscopist-blinded, randomised, controlled study. Gut 2014; 63: $125-130$

[10] Guo X, Yang Z, Zhao L et al. Enhanced instructions improve the quality of bowel preparation for colonoscopy: a meta-analysis of randomized controlled trials. Gastrointest Endosc 2017; 85: 90-97

[11] Ghevariya V, Duddempudi S, Ghevariya $\mathrm{N}$ et al. Barriers to screening colonoscopy in an urban population: a study to help focus further efforts to attain full compliance. Int J Colorectal Dis 2013; 28: 1497 1503

[12] Liu Z, Zhang MM, Li YY et al. Enhanced education for bowel preparation before colonoscopy: A state-of-the-art review. J Dig Dis 2017; 18: $84-91$

[13] Issue Information-Declaration of Helsinki. J Bone Miner Res 2017; 32: BM

[14] Wallace MB, Wang KK, Adler DG et al. Recent Advances in Endoscopy. Gastroenterology 2017: doi:10.1053/j.gastro.2017.06.014

[15] Valdovinos Andraca F, Elizondo-Rivera J, de la Torre Bravo A et al. Segundo consenso mexicano para la Enseñanza de la endoscopia gastrointestinal. Endoscopia 2015; 27: 14-24

[16] Faulx AL, Lightdale JR, Acosta RD et al. Guidelines for privileging, credentialing, and proctoring to perform $\mathrm{Gl}$ endoscopy. Gastrointest Endosc 2017; 85: $273-281$

[17] Lebwohl B, Kastrinos F, Glick M et al. The impact of suboptimal bowel preparation on adenoma miss rates and the factors associated with early repeat colonoscopy. Gastrointest Endosc 2011; 73: 1207-1214

[18] Parmar R, Martel M, Rostom A et al. Validated Scales for Colon Cleansing: A Systematic Review. Am J Gastroenterol 2016; 111: 197 204

[19] Lai E], Calderwood AH, Doros G et al. The Boston bowel preparation scale: a valid and reliable instrument for colonoscopy-oriented research. Gastrointest Endosc 2009; 69: 620-625

[20] Gurudu SR, Fry LC, Fleischer DE et al. Factors contributing to patient nonattendance at open-access endoscopy. Dig Dis Sci 2006; 51: $1942-1945$

[21] Childers RE, Laird A, Newman L et al. The role of a nurse telephone call to prevent no-shows in endoscopy. Gastrointest Endosc 2016; 84: $1010-1017$

[22] Lee CS, McCormick PA. Telephone reminders to reduce non-attendance rate for endoscopy. J R Soc Med 2003; 96: 547 - 548

[23] Chan WK, Saravanan A, Manikam J et al. Appointment waiting times and education level influence the quality of bowel preparation in adult patients undergoing colonoscopy. BMC Gastroenterol 2011; 11 : 86

[24] Seo EH, Kim TO, Park M] et al. Optimal preparation-to-colonoscopy interval in split-dose PEG bowel preparation determines satisfactory bowel preparation quality: an observational prospective study. Gastrointest Endosc 2012; 75: $583-590$ 
[25] Calderwood AH, Schroy PCIII, Lieberman DA et al. Boston Bowel Preparation Scale scores provide a standardized definition of adequate for describing bowel cleanliness. Gastrointest Endosc 2014; 80: $269-276$

[26] Shaikh AA, Hussain SM, Rahn S et al. Effect of an educational pamphlet on colon cancer screening: a randomized, prospective trial. Eur J Gastroenterol Hepatol 2010; 22: 444 -449

[27] Shaukat A, Rector TS, Church TR et al. Longer withdrawal time is associated with a reduced incidence of interval cancer after screening colonoscopy. Gastroenterology 2015; 149: 952 - 957

[28] Kashiwagi K, Inoue N, Yoshida T et al. Polyp detection rate in transverse and sigmoid colon significantly increases with longer withdrawal time during screening colonoscopy. PLoS One 2017; 12: e0174155

[29] Asadzadeh AH, Nazemalhosseini ME, Ashtari S et al. Polyp detection rate and pathological features in patients undergoing a comprehensive colonoscopy screening. World J Gastrointest Pathophysiol 2017; 8: $3-10$

[30] Chokshi RV, Hovis CE, Hollander T et al. Prevalence of missed adenomas in patients with inadequate bowel preparation on screening colonoscopy. Gastrointest Endosc 2012; 75: 1197-1203

[31] de Lascurain-Morhan E. [Prevalence of adenomas and carcinomas of the colon. Results of the rectosigmoid exam]. Rev Gastroenterol Mex 2001; 66: $131-136$
[32] Yadlapati R, Gawron A, Keswani RN. Patient satisfaction does not correlate with established colonoscopy quality metrics. Am J Gastroenterol 2014; 109: 1089-1091

[33] Ekkelenkamp VE, Dowler K, Valori RM et al. Patient comfort and quality in colonoscopy. World J Gastroenterol 2013; 19: 2355 - 2361

[34] Bretthauer M, Aabakken L, Dekker E et al. Reporting systems in gastrointestinal endoscopy: Requirements and standards facilitating quality improvement: European Society of Gastrointestinal Endoscopy position statement. United European Gastroenterol ] 2016; 4: $172-176$

[35] Hancock KS, Mascarenhas R, Lieberman D. What can we do to optimize colonoscopy and how effective can we be? Curr Gastroenterol Rep 2016; 18: 27

[36] Lee YJ, Kim ES, Choi JH et al. Impact of reinforced education by telephone and short message service on the quality of bowel preparation: a randomized controlled study. Endoscopy 2015; 47: 1018-1027

[37] Patel AR, Kessler ], Braithwaite RS et al. Economic evaluation of mobile phone text message interventions to improve adherence to HIV therapy in Kenya. Medicine (Baltimore) 2017; 96: e6078

[38] Dube C, Rostom A. Acquiring and maintaining competency in gastrointestinal endoscopy. Best Pract Res Clin Gastroenterol 2016; 30: $339-347$

[39] Macias Angeles YR, Saraiba Reyes M, Tejada Garcia RA et al. Comparación de la efectividad de 2 esquemas de preparación intestinal para colonoscopia en el Hospital General de México. Endoscopia 2015; 27: $98-103$

\section{CORRECTION}

Marisol Gálvez, Angel Mario Zarate, Hector Espino et al. A short telephone-call reminder improves bowel preparation, quality indicators and patient satisfaction with first colonoscopy.

Endoscopy International Open 2017; 05: E1172-E1178

DOI: $10.1055 / \mathrm{s}-0043-117954$

A word was missing in $>$ Table 5. "Hyperplastic" was added. This was corrected in the online version on December 6, 2017. 